\title{
El proceso político en la formulación de la política pública de integración y participación de las mujeres en las Fuerzas Armadas: el caso del Ejército de Chile, 1974 -2009.
}

The political process in the formulation of public policy integration and participation of women in the armed forces: the case of the Chilean Army, 1974 -2009.

\section{Resumen}

Daisy Penrroz Maldonado*

El artículo analiza el proceso político en la formulación de la política pública de integración y participación de las mujeres en las Fuerzas Armadas, a partir de una aproximación politológica se plantea que en el caso del Ejército de Chile, fue un proceso complejo que se desarrolló desde consideraciones ético-morales planteadas al interior del Ejército hasta convertirse en un debate público basado en argumentaciones políticas. En esa línea, cabe reconocer tres momentos claves: La incorporación por complemento en la Escuela de los Servicios Auxiliares Femeninos del Ejército entre 1974 y 1995; los avances en la integración en la Escuela Militar Bernardo O'Higgins entre 1995 y 2002 y; la generación de la política pública vía ejecutivo entre 2002 y 2009. La metodología se realiza a partir de un enfoque cualitativo basado en técnicas de análisis documental.

Palabras clave: Ejército de Chile- incorporación - políticas públicas - integración mujeres.

\begin{abstract}
The article analyzes the political process in the formulation of public policy integration and participation of women in the Armed Forces, from a political science approach arises in the case of the Chilean Army, was a complex process that ran from ethical and moral considerations raised within the army into a public debate based on political arguments. In that vein, it should recognize three key moments: Incorporating complement the School of Army Female Auxiliary Services between 1974 and 1995; advances in integration into the Bernardo O'Higgins Military School between 1995 and 2002; the generation public policy executive road between 2002 and 2009. The methodology was made from a qualitative techniques based on documentary analysis approach.
\end{abstract}

Key words: Chilean Army - incorporation- public politics - integration - women.

Fecha de recepción: 19 de septiembre 2015

Fecha de aprobación: 12 de abril 2017

\footnotetext{
* Magister (C) en Historia, mención Historia de Chile. Licenciada en Historia y Profesora de Estado en Historia y Ciencias Sociales de la Universidad de Santiago de Chile.
} 


\section{Introducción}

Latinoamérica, registra desde la década de 1990 una mayor visibilidad de la participación de las mujeres en el ámbito militar, la cual se vincularía con los procesos democratizadores de la región (Avelar, Renata, 2010). Sin embargo, es posible rastrear con antelación su incorporación en varios países ${ }^{1}$. En Chile, la incorporación de mujeres se inició en la década de 1970 dentro de las fuerzas terrestre y aérea, proceso que se replicaría en la región sudamericana a partir de 1980 y $1990^{2}$.

Es así, como el 19 de agosto de 1974 por Decretos Supremos DOE. II № 317 y 318 se creó la Escuela del Servicio Auxiliar Femenino del Ejército (E.S.A.F.E.), lo que permitió el ingreso de mujeres como parte del cuerpo profesional al Ejército de Chile. Durante la década de 1990, y de acuerdo al Plan de Modernización del Ejército de Chile, se dispuso el cierre de dicha Escuela con el propósito de que las mujeres egresaran de las Escuelas Matrices como parte del cuerpo comando. Posteriormente, el Ministerio de Defensa integró un enfoque de género en la Política de Integración y Participación de las Mujeres en las Fuerzas Armadas y de Orden y Seguridad (2005), la que se plasmó en una Separata de Actualización del Libro de la Defensa Nacional. En ella, se perfiló una serie de criterios comunes para todas las Fuerzas Armadas en su conjunto, de modo de reglamentar e institucionalizar la igualdad de oportunidades en la carrera militar ${ }^{3}$.

En base a lo anterior, el tiempo que ha transcurrido entre 1974 a 2009, permite identificar diversos momentos en la formulación de las políticas públicas que reglamentaron la situación de las mujeres al interior del Ejército. La presente investigación tiene por objetivo analizar la formulación de las políticas públicas en torno a la participación femenina en el Ejército, desde un punto de vista del análisis del proceso político. En este sentido, la pregunta de investigación radica en ¿Cómo se desarrolló el proceso político que llevó a la formulación de la política pública de integración y participación de las mujeres en las Fuerzas Armadas en 2005 al interior del Ejército de Chile?. Esta pregunta directriz contiene a su vez otras preguntas: ¿Cuál fue el origen que tuvo esta política pública? ¿Qué procesos se desarrollaron para que el tema de la participación de las mujeres en el Ejército se transformara en un problema que debió ser abordado por el aparato ejecutivo del Estado? ¿Cuáles son los actores que jugaron roles relevantes en este proceso? ¿Qué circunstancias favorecieron o dificultaron que esta iniciativa se transformara en política pública?

\footnotetext{
${ }_{1}^{1}$ Colombia (1976), El Salvador (1971), Guatemala (1967), Paraguay (1932), Ecuador (1956), Chile (1974)

2 Argentina (1982), Bolivia (1982), Brasil (1992), Perú (1997).

${ }^{3}$ Siguiendo esta política pública, en agosto de 2009, el sector Defensa puso en marcha el Plan de Acción Nacional, para la implementación de la Resolución 1325, trabajo que fue coordinado por los ministerios de Defensa, Relaciones Exteriores y Servicio Nacional de la Mujer.
} 
La justificación de la problematización, se realiza sobre la base de cuatro consideraciones. En primer lugar, el proceso de incorporación femenina al ámbito militar, pues resulta peculiar que instituciones castrenses desarrollen procesos de incorporación femenina, considerando que la guerra ha sido función por excelencia de los hombres, siendo las mujeres excluidas del manejo de las armas (Perrot, Michelle, 1997). No obstante, a pesar que este proceso supone una creciente incorporación de mujeres en los ejércitos de la Organización del Tratado del Atlántico Norte, existen resistencias culturales en países, tales como, Polonia y Turquía, cuyos porcentajes son inferiores al $5 \%$ de mujeres en las Fuerzas Armadas (Costantini, Pablo, 2008).

En segundo lugar, el caso de estudio del Ejército de Chile resulta interesante, pues fue pionero en el proceso de militarización femenina en la región sudamericana, siendo incluso el primero en incorporar mujeres como parte del cuerpo comando en 1995, lo que replicó posteriormente la Armada y Fuerza Aérea, 2007 y 2000 respectivamente.

En tercer lugar, pues constituye un debate en desarrollo dentro de los procesos de democratización en América Latina. El debate sobre modernización y reforma al sector de Defensa ha atendido en primera instancia a los ámbitos políticos institucionales dirigidos a ejercer el control y liderazgo civil sobre las FF.AA. Con posterioridad, la estabilidad democrática en los países del continente, abriría paso a un nuevo periodo de reformas institucionales en el sector defensa y en las FF.AA., en ámbitos tales como la economía, la justicia militar, el servicio militar, el presupuesto, los Derechos Humanos y la participación de la mujer en las FF.AA (Tellería, Loreta, 2008). En este último debate se inserta el presente estudio. Por último, debido al carácter contemporáneo del proceso, existen escasos estudios sobre el tema, lo que denota un vacío bibliográfico al respecto, pues constituye un estudio en pleno desarrollo.

En relación a la hipótesis central, se propone que el proceso político en la formulación de la política pública de integración y participación de las mujeres en el Ejército de Chile fue un proceso complejo que se desarrolló desde consideraciones ético-morales planteadas al interior del Ejército hasta convertirse en un debate público basado en argumentaciones políticas. En esa línea, cabe reconocer tres momentos claves:

a) La incorporación como parte del cuerpo profesional en la ESAFE en 1974 hasta el año 1995, cuando el contingente femenino pasó a desarrollar su proceso formativo en la Escuela Militar, se caracterizó por ser un tema que careció de políticas públicas, restringiéndose al interior de la institución, primando consideraciones ético-morales representadas por el Comandante de entonces Augusto Pinochet Ugarte.

b) El paso de la mujer al cuerpo comando en 1995 en la Escuela Militar Bernardo O'Higgins hasta 2002 representó avances en la carrera militar y el comienzo de un diálogo con los marcos conceptuales elaborados por Naciones Unidas. No obstante, las 
consideraciones ético-morales y de racionalidad económica al interior de la institución militar siguieron primando, coincidiendo la Comandancia en Jefe de Ricardo Izurieta Caffarena con la anterior de Augusto Pinochet, en cierta subjetividad en la identificación del tema de la participación de las mujeres en el Ejército.

c) Las intervenciones de política pública se producen 30 años después de la incorporación de la mujer como parte del cuerpo profesional. Por un lado, entre 2002 y 2009, la pugna de intereses confluyó en la formulación de una política pública vía ejecutivo, cuyo origen se sustentó en la necesidad de atender a las demandas e intervenciones de la ONU, respondiendo al debate internacional propugnado por dicho organismo 10 años atrás en razón del acceso y toma de decisiones de poder en las mujeres bajo la lógica de la equidad de género. Por otro, la actuación política del Comandante Juan Emilio Cheyre y su reconocimiento público al tema de los DDHH y, posteriormente, la motivación presidencial de Michelle Bachelet y su impulso al enfoque de género, permitieron la reglamentación de la situación de las mujeres al interior de la institución (2005) y la puesta en marcha del Plan de Acción Nacional (2009) para la implementación de la Resolución 1325, trabajo coordinado por los ministerios de Defensa, Relaciones Exteriores y el Servicio Nacional de la Mujer.

El enfoque teórico de este estudio se sustenta en una aproximación politológica, pues los valores jugarían un rol central en el proceso de la política pública, las cuales no son intervenciones neutras, sino que surgen de un proceso político y se deciden políticamente, siendo decisiones, con frecuencia, más bien incrementales y que responden a factores críticos de la contingencia política [...] en definitiva, sería resultado de un conjunto de interacciones entre actores de poder, en las que los valores, coyunturas, instituciones y cultura también juegan un rol (Olavarría, Mauricio, 2010). Por otro lado, se sustenta en el llamado estudio de caso, estrategia preferida cuando la pregunta de investigación es acerca de "cómo" o "por qué", cuando el investigador tiene poco control sobre los eventos y cuando el foco de investigación es un fenómeno contemporáneo de la vida real (Olavarría, Mauricio, 2010). Si bien existen elementos comunes y generales en el proceso de incorporación femenina a las Fuerzas Armadas, cada rama de la Defensa presenta sus propias dinámicas internas, ritmos y especificidades en relación al tema, motivo por el cual se analiza el caso del Ejército.

Se considera el concepto de transversalización de la perspectiva de género, a partir del cual la participación femenina cobraría relevancia a través de la intervención del Consejo de Seguridad de las Naciones Unidas en el tema, y de una activa política plasmada en Convenciones, Estatutos y Declaraciones. Quizás, la más importante de todas haya sido la celebrada en Beijing en el año 1995, que trasladó el centro de la atención al concepto de género, reconociendo que toda estructura de la sociedad y todas las relaciones entre hombre y mujeres, tenían que ser reevaluadas para potenciar plenamente el papel de la mujer. Concretamente, se estableció el concepto de transversalización de la perspectiva 
de género como estrategia principal, que conduciría a la concreción de la equidad de género. Esta perspectiva será la que se plasme en las políticas públicas del 2005 y del 2009.

Dentro de los aportes más relevantes que se han hecho sobre el tema de investigación es posible identificar tres líneas fundamentalmente. La primera, correspondería a estudios desarrollados en el extranjero, que dan cuenta de la incorporación de mujeres al área militar a partir del siglo XIX hasta llegar al tema de las Operaciones de Paz en el siglo XXI. Una segunda línea de investigación, abarcaría los estudios desde una perspectiva politológica e institucional $y$, una tercera línea investigativa que ha desarrollado los estudios desde la perspectiva de género. Estudio aludirá a la segunda y tercera línea.

En esa línea, es posible reconocer tres principales factores que llevan a la integración de las mujeres a las Fuerzas Armadas. Primero, la democracia que cada vez exige mayor igualdad de oportunidades para los ciudadanos. Segundo, el cambio en la concepción de las nuevas guerras, avances tecnológicos y administrativos. Y tercero, el factor psicosocial, la percepción de los agentes sobre la función de los militares, donde incluye el prestigio de la profesión militar y la legitimidad castrense (Kalil, Suzeley, 2005).

No obstante, habría que tener cuidado respecto al primer factor, porque esa democracia que cada vez exige mayor igualdad de oportunidades, tuvo que resolver otros problemas antes de dar cabida a los temas de género. En Sudamérica, las reformas al sector defensa y FF.AA. se caracterizaron por el resultado de las transiciones políticas a la democracia. Es así como en una primera etapa de modernización o reforma del sector defensa en América del Sur, se dio prioridad a los ámbitos políticos institucionales dirigidos a ejercer el control y liderazgo civil sobre las FF.AA. Con posterioridad, la estabilidad democrática en los países, abriría paso a un nuevo periodo de reformas institucionales en el sector defensa y en las FF.AA., en ámbitos tales como la economía, la justicia militar, el servicio militar, el presupuesto, los Derechos Humanos y la participación de la mujer en las FF.AA (Tellería, Loreta 2008), pero ¿cómo se dio esta "estabilidad democrática" en Chile?. Respecto al segundo factor, habría que considerar en qué medida los cambios operados a nivel internacional ejercieron influencia en Chile.

La incorporación de la mujer a las Fuerzas Armadas de los países de América Latina, no ha sido un proceso lineal. En cada país es posible enumerar diversos factores históricos, sociales, políticos y culturales que han dado una impronta particular a cada caso, de modo que constituyen procesos heterogéneos, dependientes de la imagen de la mujer en cada sociedad y de la trayectoria histórica de los países en particular. Sin embargo, los hechos coyunturales conforman un importante determinante en la incorporación de las mujeres en las fuerzas armadas, pudiendo distinguirse grandes etapas que la mayoría de ellos comparten. Éstas estarían marcadas por el debate de si son parte de la carrera militar en el sentido más tradicional de la misma (Donadio y Mazzota, 2009). 
En una primera etapa, la presencia de la mujer entre los soldados habría ocurrido más por necesidad y en determinados contextos históricos. Esta participación femenina, habría estado dada por la necesidad de las instituciones armadas de cubrir vacantes de personal en contextos históricos particulares. Por ejemplo, las guerras de Independencia, donde la mujer se desempeñaba en funciones auxiliares, como administrativas y de sanidad. Sin embargo, una vez finalizado los períodos de conflicto, las mujeres eran desligadas de sus servicios y retornaban a sus actividades tradicionales (Donadio y Mazzota, 2009).

Esta incorporación no respondía a una política de integración, la cual comenzaría como parte del proceso de profesionalización de las instituciones armadas de la región latinoamericana, que habría comenzado a fines del siglo XIX e inicios del siglo XX, bajo los modelos europeos de ejércitos profesionales. En esta segunda etapa, la mayoría de las mujeres fueron incorporadas al cuerpo profesional. Ellas fueron asimiladas como especialistas y desarrollaron sus funciones en las distintas organizaciones militares como abogadas, médicas, enfermeras, administradoras, profesoras, psicólogas, por mencionar algunas. La tercera etapa en el proceso de incorporación femenina a las fuerzas armadas latinoamericanas, comenzaría a desarrollarse a partir de la década de los noventa, y con los procesos de democratización emprendidos por la región durante este período. Esta etapa, estaría marcada por el acceso a la carrera militar propiamente tal, pasando las mujeres a conformar parte del cuerpo comando.

Este estudio, tiene especial cuidado con la tercera etapa, pues intenta desarrollar un análisis que considere las especificidades respecto al Ejército de Chile, pues como señala Michelle Perrot, refiriéndose al ingreso de las jóvenes a las escuelas militares, habría que matizar, pues "el porcentaje de mujeres seguiría siendo débil, y convendría distinguir los rangos jerárquicos, el tipo de armas y de técnicas" (Perrot, Michelle, 1997: 136). En Chile, realizar un estudio sobre la participación femenina en las Fuerzas Armadas sería un tema difícil, porque está cargado de subjetividades y de opiniones contrapuestas (Arancibia, Claudia y Tatiana Milstein, 2002). No obstante, el aspecto positivo de la mayor incorporación de las mujeres a las Fuerzas Armadas se destaca como parte de una evolución histórica que estaría encontrando un mayor grado de igualdad y legitimidad de las mujeres al interior de dicha institución. Por otro lado, se debe considerar que aún persisten límites para el desempeño de la mujer militar en ciertas armas o escalafones, no obstante las dinámicas particulares con que se han implementado las políticas para contribuir al logro de la igualdad y la existencia de liderazgos femeninos en lugares clave de la toma de decisiones en esta materia, han influido en la incorporación en igualdad de la mujer en las Fuerzas Armadas (Castrillón, Liliana y Pía Von Chrismar, 2013).

Si bien las contribuciones académicas de la Red de Seguridad y Defensa de América Latina (RESDAL) y la Academia de Estudios Políticos y Estratégicos (ANEPE) aportan antecedentes para la comprensión del tema, han tomado los acontecimientos dentro de 
esquemas explicativos que los colocan en correlación con regularidades, tales como la estructura sociopolítica experimentada por la región sudamericana, tendiendo a encasillarla bajo el contexto transicional democrático de la década de los noventa, no dando cuenta que la incorporación se inicia en otro contexto político a saber, el de implantación de regímenes militares de la década de los setenta y ochenta en el cono sur americano.

La estrategia metodológica de tipo interpretativa se realiza a partir de un estudio de caso y enfoque cualitativo, basado en técnicas de análisis documental de fuentes primarias y secundarias. Las primeras, provienen de documentos oficiales tales como los planes de estudio dirigidos a hombres y mujeres entre los años 1974 y 2003 -disponibles en la Escuela Militar-, declaraciones, clases magistrales, intervenciones, boletines, órdenes de comando, discursos, memoriales, decretos supremos y resoluciones -disponibles en el Departamento de Historia Militar- y revistas de la institución -Academia de Guerra-. Las segundas, están basadas en bibliografía contemporánea que se ha escrito al respecto.

No obstante, cabe señalar la dificultad que reviste acceder a estas fuentes, pues no se encuentran disponibles en bibliotecas públicas de fácil acceso, siendo más bien, resguardas en las mismas instituciones y bibliotecas militares con carácter, incluso, de fuentes reservadas. Se visitó el Departamento de Historia Militar del Ejército, la Academia de Guerra, el Museo Histórico y Militar de Chile y, la Escuela Militar del Libertador Bernardo O'Higgins, además de solicitar un permiso especial antes las autoridades de cada lugar, hay que considerar como limitación de la muestra que el material proporcionado está previamente seleccionado y autorizado por los encargados respectivos, de modo que constituyen discursos hegemónicos de los actores con poder dentro del Ejército. Así también, una limitación de la técnica utilizada, radica en la falta de entrevistas en profundidad a mujeres y hombres que vivieron estas experiencias exceptuando algunas entrevistas realizadas en las revistas institucionales a las mujeres, sin embargo son revistas que provienen del discursos oficial-, aspecto que se sugiere ser realizado en investigaciones futuras. A través de internet fue posible acceder ampliamente a los Memoriales, los cuales contienen artículos escritos por las mismas autoridades militares, siendo abordados aquellos temas que son relevantes para la institución; fueron revisados memoriales desde el año 1971 hasta el año 2006, junto con discursos de los Comandantes -disponibles en la página oficial de la Escuela Militar-. Al indagar en ellos, las vastas ausencias discursivas respecto de la mujer en el Ejército dejan un vacío difícil de llenar, pero a su vez, da luces de la concepción de género de la oficialidad.

La presentación de la evidencia se presenta en tres capítulos a saber: el primero, caracteriza la incorporación femenina a la ESAFE entre 1974 y 1995, para así dar cuenta del contexto, actores y motivos por los cuales se incorporan mujeres al Ejército, así como del tipo de integración en este momento inicial. El segundo capítulo, analiza la 
participación de las mujeres como parte del cuerpo comando, lo cual permitirá comparar dos momentos en la participación de las mujeres en el Ejército, antes en la ESAFE y a partir de 1995 en la Escuela Militar Bernardo O'Higgins. Se pretende dar cuenta de cómo cambió la visión del problema y qué cambios se produjeron en la intervención del Estado entre 1995 y 2002. Finalmente, en el tercer capítulo el énfasis está en describir los rangos centrales de la relación entre el gobierno y el Ejército, considerando factores internacionales que catalizaron la promulgación de políticas públicas en el área de la Defensa y cómo éstas se implementaron en la realidad chilena entre 2002 y 2009. De esta forma, se pretende generar conocimiento sobre un tema contingente sobre el cual las políticas públicas siguen estructurándose, de modo de instar a ampliar el análisis a las otras ramas armadas, lo cual será labor de futuras investigaciones.

\section{Los Hechos En El Proceso De Integración De La Mujer Al Ejército 1974 - 2009}

En una primera etapa, la presencia de la mujer entre los soldados habría ocurrido más por necesidad y en determinados contextos históricos. Esta participación femenina, habría estado dada por la necesidad de las instituciones armadas de cubrir vacantes de personal en contextos históricos particulares. Por ejemplo, las guerras de Independencia, donde la mujer se desempeñaba en funciones auxiliares, como administrativas y de sanidad. Sin embargo, una vez finalizado los períodos de conflictos, las mujeres eran desligadas de sus servicios y retornaban a sus actividades tradicionales (Donadio y Mazzota, 2009). A partir de mediados de siglo XX, la tendencia en la región fue la incorporación de las mujeres a los cuerpos profesionales de las fuerzas armadas.

A) La Incorporación Por Complemento En La Escuela De Los Servicios Auxiliares Femeninos Del Ejército, 1974 - 1995: El 19 de agosto de 1974 por Decretos Supremos DOE. II № 317 y 318 se creó la Escuela del Servicio Auxiliar Femenino del Ejército (E.S.A.F.E.) dependiente del Comando de Institutos Militares (C.I.M.), cuyo nombre patronímico fue designado como doña "Javiera Carrera Verdugo", y el Servicio Femenino del Ejército. En esta resolución firmaron Oscar Bonilla Bradanovic, General de División y Ministro de Defensa Nacional y, Augusto Pinochet Ugarte, General de Ejército y Jefe Supremo de la Nación ${ }^{4}$.

El Servicio Militar Femenino, dirigido entonces por el Comandante de Instituciones Militares Nilo Floody Buxton, anunció la puesta en marcha de dicho proyecto en 1976, señalando el carácter voluntario de éste, en donde las aspirantes serían solteras y podrían casarse después de cuatro años como subteniente, comenzando su jornada de trabajo a las seis horas con la diana y concluyendo a las veinte y un con la retreta ${ }^{5}$. Las alumnas

\footnotetext{
${ }^{4}$ Decreto Supremo DOE. II № 317, disponible en Archivo del Ejército de Chile.

${ }^{5}$ La Tercera, 1 de octubre de 1974, en Comité de Cooperación para la Paz en Chile, COPACHI, La situación general de la mujer bajo el gobierno militar de Chile, Santiago, 1975, p. 3 Citado en Javier Maravall Yáguez.
} 
Oficiales, una vez ingresadas al curso E.S.A.F.E. podían postular a las áreas de: Servicios de Ayudantía General, Intendencia, Sanidad, Material de Guerra, Transporte, Bienestar Social e Instructoras de Educación Física. A su vez, las alumnas Clases dentro de cada área de los servicios, anteriormente enunciados, podían postular a las especialidades como Suboficiales, en Ayudantía General: como registradora, auxiliar de dactilografía y secretaría, comunicaciones telefónicas y telegráficas y, estafetas. En Intendencia: como auxiliar dietista o nutricionista, operadoras de lavandería, operadoras de vestuario, operadoras de confección de alimentos. En Sanidad: como auxiliar de sanidad y de sanidad dental, veladoras de hospitales militares, auxiliares de rayos X. En Material de Guerra: como operadoras de estaciones de servicio. En Transporte: como conductoras de vehículos motorizados. En Bienestar Social: como auxiliares de asistente social, rehabilitación y auxiliares de párvulos.

Desde el punto de vista de una mujer militar, que ingresó por entonces a la E.S.A.F.E., "la idea de este proyecto que partió un poco como experimento, era formar mujeres para que participaran no en la guerra, sino en las labores de administración del Ejército" ${ }^{6}$. En este sentido, las razones de las mujeres para incorporarse al ámbito militar fueron variadas. Por un lado, la actual teniente coronel María Isabel Alegría señaló que cuando apareció la posibilidad de que las mujeres pudieran ingresar como uniformadas al Ejército, entró por curiosidad, porque nadie sabía nada, constituyéndose en un reto, una opción distinta, la cual complementó una parte útil de ella. Por otro lado, en una entrevista realizada a la Mayor Leticia Pérez, señaló que sus razones fueron prácticas, ya que llevaba seis años casada con un militar, sin hijos y titulada de asistente social. No lograba encontrar empleo, por lo que decidió rendir el examen de admisión y entrar al Ejército (Zalaquett, Cherie, 2009).

Además de ejercer dentro de las distintas instalaciones militares educacionales o permanecer en la E.S.A.F.E. como instructoras de futuros cursos, podían ser destinadas como instructoras de la Institución a las Compañías del S.A.F.E. que funcionaban a partir de 1974 en Iquique, Antofagasta, Santiago, Concepción, Valdivia y Punta Arenas (Boletín del Ejército, 1974). Vistos los resultados obtenidos con el desarrollo del primer curso del Servicio Auxiliar Femenino del Ejército, se resolvió realizar durante el año 1975 un segundo curso para la formación de Monitoras de la E.S.A.F.E. A través de una Circular Reservada emitida por el Comandante del Comando de Institutos Militares, se dictaron las correspondientes instrucciones a saber (Circular del Ejército, 1974). Los cursos funcionarían entre el 3 de marzo al 17 de diciembre con 50 vacantes para el de Oficiales y entre el 14 de abril y el 17 de diciembre con 100 vacantes para el de Clases; el grado de egreso seguiría siendo el mismo, vale decir, Subtenientes y Cabos segundos, mientras que el régimen de estudios sería de internado con salida sólo los fines de semana.

El ideario de mujer bajo la dictadura militar (1973 - 1990), Universidad Autónoma de Madrid, Revista Electrónica de Historia: Pensamiento Crítico №4, Noviembre, 2004.

"Entrevista revista "Hoy" № 2 Cuestión de Honor, pp. 14 -17, 21 de septiembre 1998. 
No obstante, las oficiales y suboficiales de la E.S.A.F.E. no pudieron estudiar en las Academias de Guerra ni Politécnica. Al respecto, en una entrevistada realizada por Cherie Zalaquett, señaló que la diferencia de preparación intelectual se reflejaba en sus sueldos, ya que por no ser oficiales de Estado Mayor ganaban menos que sus pares masculinos, aunque si tenían estudios superiores, percibían mayor salario que un militar en el mismo puesto sin título universitario (Zalaquett, Cherie, 2009).

B) Avances En La Integración En La Escuela Militar Bernando O’higgins Riquelme, 1995 2002: Durante la década de los noventa, y de acuerdo al Plan de Modernización del Ejército de Chile, se fueron produciendo cambios para las integrantes femeninas uniformadas. Es así como en el año 1995, se dispuso el cierre de la Escuela del Servicio Femenino Militar con el propósito de que las Oficiales y Suboficiales egresaran de las Escuelas Matrices, incorporándose de esta forma al cuerpo comando del Ejército.

Tabla 1. Total alumnos Escuela Militar Masculinos y Femeninos, 1996.

\begin{tabular}{|l|l|l|}
\hline Nivel & Cantidad Cursos & Cantidad Alumnos \\
\hline I.A.E & 12 & 280 \\
\hline II. A.E & 10 & 200 \\
\hline III. A.E & 07 & 210 \\
\hline IV. A.E & 06 & 180 \\
\hline V. A.E & 08 & 162 \\
\hline C.A.O.F.M. & 01 & 20 \\
\hline C.A.O.F.M. & 01 & 19 \\
\hline
\end{tabular}

Fuente: Plan de Estudios Ejército de Chile. En Comando de Institutos Militares Escuela Militar, 1996.

A pesar que en términos de cifras la presencia de la mujer fue minoritaria respecto de sus pares masculinos, los cambios dentro de los cuales se produce esta incorporación resultan importantes. El ingreso de la mujer a la Escuela Militar entre 1996 y 1998, consistió en dos años de educación, perteneciendo al Escalafón de Oficial de Servicio Femenino Militar, en una educación que fue separada de los hombres, teniendo asignaturas diferentes según su especialidad. Por un lado, fueron especialistas en Finanzas con mención en Informática y Computación y, por otro, especialistas en Administración de Personal con mención en Relaciones Públicas. A diferencia de los hombres, los cuales debían en los dos primeros años de escuela terminar la enseñanza media, las mujeres ingresaban con la enseñanza media completa. En términos de acceso al poder, el D.F.L. № 1 “Estatuto del Personal de las FF.AA." de 1997, estableció el tiempo de requisito en los grados para las Oficiales, extendiéndose a 30 años de servicios válidos 
para el retiro, creándose además la vacante de Coronel como Oficial de Línea en el Escalafón del Servicio Femenino Militar.

Una vez que asume la Comandancia en Jefe Ricardo Izurieta Caffarena, éste amplió los alcances del Plan Alcázar de su antecesor, Augusto Pinochet, y proyectó el desarrollo de dicho plan considerando clave la subordinación al poder civil, elemento necesario para construir una institución profesional. Se pretendía implementar cambios en la planificación docente y la formación de la oficialidad, para acomodarlos a los nuevos sistemas organizacionales de las unidades operativas. En este sentido, el proceso de modernización modificó aspectos referidos a la integración de la mujer en el Ejército:

Las mujeres chilenas podrán ingresar en el ejército como soldados y llegar hasta General, e incluso integrar el Alto Mando del Ejército [...] Estamos absolutamente encantados con la posibilidad [...] A partir del próximo año se reimplantará el servicio militar voluntario para las mujeres [...] En 10 o 15 años las mujeres podrán llegar al rango de General y hasta al Alto Mando, aunque no a la Comandancia en Jefe; eso el tiempo lo dirá. (Diario La Nación, Sábado 15 de agosto de 1998)

El anuncio, se erigía sobre la base de que las mujeres podrían acceder a nuevos escalafones que antes no les estaban permitidos, no obstante, la advertencia de que no podrían ser Comandantes en Jefe, se realizó sobre la consideración de que para llegar a ese grado había que, por un lado, realizar un curso de Estado Mayor -el cual por entonces se encontraba vedado para las mujeres- $y$, por otro lado, pertenecer previamente al Escalafón de las Armas, escalafón al cual aún no podían acceder las mujeres. Así, mediante Boletín Institucional ${ }^{7}$ de fecha 12 de agosto del mismo año, se entregó información referida al Servicio Femenino Militar:

Con el propósito de dar un efectivo paso en el proceso de modernización institucional, que permita el pleno desarrollo profesional del personal femenino, el Sr. Comandante en Jefe del Ejército, TGL Ricardo Izurieta Caffarena, el próximo 14 de agosto en una ceremonia que se efectuará en el Aula Magna de la Escuela Militar, con motivo de celebrarse el Día del "Servicio femenino Militar", firmará cuatro órdenes de Comando referidas a lo siguiente: Escalafonamiento de Oficiales Femeninos, rol del Servicio Femenino Militar, autorizar el matrimonio entre personal militar, llamados al servicio militar femenino [...]. (Gutiérrez y Maceratta, 2010)

Se señaló que a partir del primero de enero del año 1999, las diferentes promociones de Oficiales (F) egresarían de los escalafones de Servicio de Material de Guerra, Servicio de Intendencia y Servicio Femenino Militar (administración de personal), según vacantes

\footnotetext{
${ }^{7}$ Boletín Institucional, 12 de agosto de 1998, Edición año XXIV, №6708/38 Emitida por el Departamento Comunicacional del Ejército.
} 
determinadas por la Dirección del Personal del Ejército, escalafones que en términos de acceso al poder, le permitirían llegar a ser Generales de Brigada. Hacia el año 2001, se produjo una rearticulación del sistema educativo en función de los crecientes niveles de profesionalismo castrense. Así, en febrero del mismo año, ingresó la primera promoción de futuras oficiales femeninas, las cuales permanecerían durante cuatro años en la Escuela, al igual que el personal masculino.

C) Militares Y Gobierno En La Generación De La Política Pública Vía Ejecutivo, 2002 2009: Hacia principios del siglo XXI, un nuevo concepto rector del proceso de modernización cobraría relevancia, favoreciendo una gradual inclusión de las mujeres en el ámbito castrense. A partir del llamado militarismo profesional participativo, se fueron ampliando los ámbitos de desempeño del personal femenino. A su vez, se abrió la posibilidad de adquirir crecientes grados de poder, en tanto sus grados serían los mismos existentes para el género masculino, según el escalafón que cursara.

Es así como el Mando Institucional y la influencia del proceso de modernización, llevó a realizar cambios en la institución para el personal femenino militar, permitiendo el ingreso del personal femenino a las Armas de Apoyo de Combate, tales como Artillería, Ingenieros y Telecomunicaciones, con la misma línea de carrera y posibilidades de desarrollo profesional que el personal masculino, protocolizado en la Orden Comando del 29 de octubre de 2002. El Comandante en Jefe de la institución Juan Emilio Cheyre, informaba que a partir del año 2003, las mujeres podrían ingresar a la Academia de Guerra, luego al Estado Mayor y, eventualmente, alcanzar la Comandancia en Jefe. A partir de ese año, las mujeres que ingresaron al Ejército de Chile podrían hacer carrera y alcanzar, al igual que los hombres, el grado de general de Ejército, pues hasta ese momento, las mujeres sólo podían aspirar al grado de Coronel después de 30 años de servicio, o bien, al grado de General de Brigada si pertenecía al escalafón de Material de Guerra o Intendencia.

De esta manera, el Ejército acogió una sugerencia formulada por Naciones Unidas, que solicitó a los países miembros que permitieran el ingreso de las mujeres a las Fuerzas Armadas y a los más altos rangos. Así, la participación femenina cobró relevancia a través de la intervención del Consejo de Seguridad en el tema, y de una activa política plasmada en Convenciones, Estatutos y Declaraciones. En la tabla adjunta, es posible observar las promociones de oficiales egresadas a partir del año 2001 desde la Escuela Militar.

Tabla 2. Promociones de Oficiales egresadas a partir del 2002 desde la Escuela Militar.

\begin{tabular}{|l|l|l|l|l|l|l|}
\hline Escalafón & 2002 & 2003 & 2004 & 2005 & 2006 & 2007 \\
\hline Artillería & 4 & 1 & 5 & 3 & 3 & 4 \\
\hline Ingenieros & 3 & 1 & 5 & 3 & 2 & 4 \\
\hline
\end{tabular}




\begin{tabular}{|l|l|l|l|l|l|l|}
\hline Telecomunicaciones & 2 & 1 & 5 & 2 & 2 & 7 \\
\hline Material de Guerra & 2 & 1 & 5 & 2 & 2 & 7 \\
\hline Intendencia & 3 & 1 & 5 & 1 & 3 & 5 \\
\hline Serv.Fem.Mil & 3 & 4 & 5 & 6 & 7 & 7 \\
\hline Total & 17 & 9 & 31 & 18 & 19 & 31 \\
\hline
\end{tabular}

Fuente: Elaboración propia en base al Plan de Estudios Ejército de Chile. En Comando de Institutos Militares Escuela Militar, 2002.

A partir de la tabla, es posible señalar que conforme avanzó el proceso de modernización del Ejército, las mujeres obtuvieron la posibilidad de asumir nuevas funciones, tales como Artillería, Ingenieros y Telecomunicaciones -Escalafón de las Armas-. La modernización del Ejército, se entendió como una rearticulación en la educación recibida por los alumnos, por lo que e partir del año 2001, la Escuela modificó su Plan de Estudios y estableció una nueva organización curricular. El Teniente Coronel Eduardo Aldunate señaló:

Una educación superior que esté sintonizada con los escenarios emergentes y que al mismo tiempo mantenga fidelidad con lo sustantivo de nuestras tradiciones y valores [...] Todos los estudios existentes sobre cómo debe una organización transitar a un nivel alto de desarrollo, e incluso un país hacia la modernización, coinciden en que ello se hace aumentando el nivel de preparación del recurso humano, como forma de elevar su productividad. (Aldunate, Eduardo, 1996)

En base a ello, el nuevo Plan de Estudios se estructuró en cuatro años profesionales de formación superior, suprimiendo los estudios correspondientes a la Educación Media, permitiendo al término del proceso, junto con el nombramiento de Oficial de Ejército, el grado académico de Licenciado en Ciencias Militares. Este proceso se inició el año 2001, con la inclusión de un Bachillerato en Ciencias Sociales, a base de 16 asignaturas científico-humanista dictadas por la Universidad Diego Portales y, a partir del año 2003, otras dos asignaturas dictadas por la Pontificia Universidad Católica de Chile. Un ejemplo concreto de esta nueva modalidad en la línea de carrera del personal femenino se reflejó en que de las 17 alféreces egresadas de la Escuela Militar el 2002, cuatro de ellas optaron por el arma de Artillería (23\%), tres por el arma de Ingenieros (18\%) y dos por Telecomunicaciones (2\%) (Mujeres en la Defensa protagonistas del Bicentenario, Ministerio de Defensa Nacional, 2010, Academia de Guerra del Ejército, p.9).

\section{La Mujer En El Ejército: Una Discusión Desde La Óptica Del Análisis De Políticas Públicas}

La incorporación de la mujer al Ejército en 1974, si bien constituyó un hito importante en cuanto su nominación como parte del cuerpo profesional, el lugar asignado a la mujer estuvo en concordancia con la concepción hegemónica de femineidad del período, no involucrándola en labores militares ligadas al ejercicio de la guerra. Las actividades que 
podía desarrollar en el Ejército no fueron más que una extensión de las labores tradicionales asignadas a la mujer en el espacio interno del hogar, esto dada las características fisiológicas y psicológicas que le asignaba la visión de género, es decir, la mujer era débil físicamente y amante de los demás. Por ello, las modalidades de estudio eran en su mayoría una continuación de sus roles tradicionales, tales como ser cuidadora y protectora de los otros, primando así consideraciones morales en su ingreso.

Los factores que explican la incorporación remiten en su generalidad a adoptar esta medida como forma complementaria a la labor desarrollada por el hombre, el cual debe quedar libre para así ocupar puestos de combate. El Comandante en Jefe del Ejército señalaba que en el año 1973, la Institución estaba reducida en personal, material y presupuesto. Desde algunos años se había privilegiado la entrega de recursos a otros sectores del país, olvidando la necesaria ecuación entre desarrollo y Seguridad Nacional. Es por esta razón que, desde ese mismo año, el esfuerzo estuvo orientado a lograr, en primer lugar, un equipamiento básico en armamento liviano moderno, tanques y artillería, y completar las dotaciones de munición. Las situaciones vecinales conflictivas, y el aumento de la actividad terrorista subversiva, habrían sido en este período las características que se consideraron como amenazas, dando origen a una planificación más completa y realista (Pinochet, Augusto, Clase Magistral, 1992).

Respecto a los apoyos de carácter administrativo y logístico Pinochet señaló que:

La reasignación de funciones permitió encontrar formas más eficientes de administración de los recursos humanos. En este empeño por mejorar el ámbito de la Administración del Personal, durante este período se concretaron los esfuerzos para que las mujeres cumplieran su Servicio Militar en roles adecuados a su condición femenina, y se integraran en calidad de Tropa, Clases, Suboficiales y Oficiales [...] Es justo recordar, hoy, que fue este esfuerzo de organización, de adquisición de medios y de entrenamientos el que hizo posible contar con las fuerzas terrestres para evitar, por la vía de la disuasión, inminentes y graves situaciones confrontacionales. (Pinochet, 1992)

Este contexto nacional en estado de permanente conflicto dio paso a una mayor participación política de las mujeres pro-dictadura al ser incorporadas en el Ejército, lo cual podría haber sido una respuesta de las autoridades para canalizar dicha participación. Como se indicó, algunas mujeres tenían vínculos familiares directos con los soldados, por lo que podría pensarse que además de un criterio moral y ético, también primó una racionalidad política. Sin embargo, en base a la evidencia se sostiene que la integración de la mujer al Ejército, respondió fundamentalmente a un factor de carácter militar y ético/sexista. Una labor femenina de apoyo que no se involucró en tareas propias de la guerra, sino más bien en ámbitos administrativos y logísticos. Reafirmando 
lo anterior, el coronel Mario Morales, director de la ESFEMIL ${ }^{8}$, precisó a El Mercurio en 1984 que:

La mujer no recibe la misma instrucción que un hombre. Y por una razón muy sencilla: su misión no es combatir (...) La función de la Escuela es formar intelectual y físicamente a las alumnas con el fin de reemplazar al hombre-combatiente en funciones como instrucción del contingente femenino, ayudantes, oficiales de personal, secretariado, dactilografía, radiooperadoras, etc. (Valenzuela, María Elena, 1987)

Los procesos acaecidos a nivel internacional irán permeando a la sociedad chilena de manera paulatina, y si bien éstos fueron reconocidos, se hacía énfasis en llevar a cabo las transformaciones necesarias en el Ejército, pero dentro de los cánones adecuados a la realidad chilena:

El mundo actual lleva a adecuar instituciones, a reestructuras organismos, a darse otras formas de relación [...] En este sentido, es que se debe enfrentar esta necesidad, pero sólo cuando se materialicen hechos que los hagan necesarios [...] en ningún caso debemos caer en la tentación de efectuar cambios guiados sólo por apreciaciones ligeras o interpretaciones antojadizas ante situaciones nuevas (Pinochet, 1992).

De lo anterior, se puede afirmar, en primer lugar, que el reconocimiento de los cambios operados a nivel internacional, condujo a que las Fuerzas Armadas y, en particular, en Ejército de Chile, bajo el mando de Augusto Pinochet Ugarte, realizaran una lectura de dichos cambios atravesada por un mirada nacional, en la cual los factores internos se superpusieran a los cambios externos.

En segundo lugar, esta mirada que privilegió la realidad interna, hacía constante advertencia sobre el peligro de caer en la "tentación de efectuar cambios guiados sólo por apreciaciones ligeras", así como en la prescindencia de ideas foráneas, por lo que el reconocimiento de los cambios operados a nivel internacional no supone necesariamente una rearticulación inmediata en las estructuras militares chilenas, de la misma forma que no lo representarían en su pensamiento y, por tanto, en la cultura militar que le asignaba roles específicos a hombres y mujeres.

En tercer lugar, se puede observar el resguardo de la independencia que posee el Ejército para llevar a cabo sus transformaciones y definir su papel, lo que da cuenta, posiblemente, de las dificultades que supuso en los primeros años de la transición la armonía entre las relaciones cívico-militares, pues se vislumbra que para el Comandante

${ }^{8}$ El primero de agosto de 1984, la E.S.A.F.E. pasó a llamarse Escuela del Servicio Femenino Militar (ESFEMIL). 
en Jefe las Fuerzas Armadas, éstas poseían una independencia y autonomía propias para realizar los cambios que considerase necesarios. Por último, se infiere el ritmo y prioridades que tendrá el proceso de modernización, ya que la necesidad de enfrentar los cambios se realizarán cuando se hayan "materializado hechos que los hagan necesarios", por tanto, un ritmo gradual marcará la adopción a las pautas internacionales, siempre en coherencia y en respuesta a la realidad nacional chilena y de las Fuerzas Armadas. En esta línea, el BGL José Carrera señaló que:

Es un hecho que la igual capacidad y responsabilidad del hombre y la mujer justifican plenamente el acceso de ésta a las funciones laborales e intelectuales; sin embargo, la completa apreciación de la mujer exige además el reconocimiento del valor de su función maternal y familiar. Desgraciadamente, no ha existido una orientación afectiva y positiva que permita a la mujer y fundamentalmente a la sociedad adaptarse a esta nueva dualidad de funciones en orden a instarla a combinar adecuadamente su función pública con su labor de madre y esposa, por constituir éste su rol específico e irreemplazable en la sociedad. Sectores influenciados por un equivocado concepto de la igualdad han olvidado que la diferencia biológica entre hombre y mujer lleva implícita una diferenciación de roles en el hogar, lo que implica reconocer y respetar el tiempo que necesita la mujer para realizar esta función. (BGL José Carrera Rivera, 1994)

Es así, como en esta etapa existía una clara división entre funciones masculinas y femeninas, ya que las modalidades de estudio abiertas para los hombres se identificaban con el combate (infantería, artillería, caballería), mientras que las funciones femeninas eran de apoyo administrativo en temas financieros, de personal, de secretaría y manejo de documentos.

Con fecha 17 de mayo de 1994 y, a través de una Orden de Comando, el Comandante en Jefe del Ejército Augusto Pinochet dispuso la reorganización del Servicio Femenino Militar, considerando:

La experiencia obtenida de la trayectoria profesional del Servicio Femenino Militar, a partir de su creación en el año 1974, y la creciente necesidad de que el personal alcance un alto nivel de especialización en el cumplimiento de la gestión administrativa y logística de la institución [...] La necesidad de integrar, definitivamente, al personal femenino a la institución dentro del proceso de modernización del Ejército [...] La necesidad de regular adecuadamente las funciones, encuadramiento y desempeño profesional del personal femenino, a partir del año 1995 [...] Lo propuesto por el estado EMGE.DPE., en el estudio de la situación actual y proyección futura del Servicio Femenino Militar. (Pinochet, 1994) 
Para cumplir con la Orden de Comando, se dispuso el traslado del personal de la Escuela Femenina Militar a la Escuela Militar Bernardo O'Higgins, para el personal de aspirantes a oficiales y, a la Escuela de Suboficiales, para las aspirantes a Clases del Ejército, quedando de esta forma disuelta la Escuela Femenina Militar en el año 1995.

En este sentido, la incorporación de las mujeres a la Escuela Militar no supone una ruptura radical respecto de su formación en la ESFEMIL, ya que en esta etapa su proceso de formación se establece sobre una diferenciación tanto de roles como funciones Oficial del Servicio Femenino Militar especialistas en Finanzas con mención en Informática y Computación, y por otro, especialistas en Administración de Personal con mención en Relaciones Públicas. Si bien bajo la Comandancia en Jefe de Ricardo Izurieta las mujeres pudieron acceder a escalafones antes vetados -Material de Guerra e Intendencia-, el Ejército al mando de Izurieta debió enfrentar la crisis más aguda del retorno a la democracia y que menoscabó las expectativas de cambio. Se puso en evidencia constantemente la tensión entre las expectativas de cambio sembradas sobre su persona al momento de asumir la Comandancia y las concesiones que debió realizar a los uniformados por la detención del general Pinochet. Estas expectativas se sustentaban en su perfil tecnocrático y modernizador, con formación y trayectoria ligado a los sectores más profesionales de la institución. De esta manera, durante el período del General Ricardo Izurieta las expectativas encontraron dificultades debido al contexto político, tanto nacional como internacional por la detención en Londres del general Pinochet, copando su agenda y sus propósitos de modernizar la institución.

Con la gestión de Cheyre, se incorporará el elemento de subordinación al ordenamiento democrático y la confianza en la implementación de la política nacional de Defensa, la que se diseñó a partir de los cambios en el proceso de la post Guerra Fría. Dentro de este contexto, los oficiales del Ejército estarían bajo el nuevo concepto de "profesionalismo militar participativo", el cual constituía un punto medio entre dos tendencias que históricamente se han dado en Chile. La primera, vivida en la década de los 30 , se caracterizó por el ostracismo y el reduccionismo militar y, la segunda, ser profesionales, pero distinguiendo en ese ser militar las capacidades existentes para hacer "lo que es propio en el ámbito de la defensa, como así también aprovechar las potencialidades existentes, sin desnaturalizar su esencia, para cumplir otras tareas de directo servicio al país", (GDE Juan Emilio Cheyre, 2005). Este concepto, destaca que si bien los uniformados pueden ampliar sus funciones a todos los ámbitos de la seguridad nacional y la proyección de Chile dentro del escenario internacional, las instituciones no debiesen ser utilizadas políticamente, pues eso no contribuía al desarrollo nacional y la cohesión (Seguel, Felipe, 2011).

En este sentido, el reconocimiento público al tema de los Derechos Humanos, realizado por el Comandante Cheyre abrió la posibilidad para que el Ejército avanzara en su proceso de modernización, entendiendo que ésta: 
No sólo apunta al desarrollo de la nueva estructura de la fuerza, a la reestructuración de los sistemas de armas e infraestructura militar, sino también al desarrollo de su personal, ya que todo lo anterior requiere de hombres y mujeres con mayor preparación y con claras opciones de desarrollo profesional. En este orden, es interesante destacar las modificaciones efectuadas a la línea de carrera de la mujer, las que hoy poseen las mismas posibilidades de desarrollo profesional que el personal masculino [...] La carrera militar femenina se definía como "una profesión con igualdad de condiciones, igualdad de desafíos e igualdad de exigencias". (Revista Armas y Servicios, 2003: 16 - 22)

Esta transformación del Ejército, en uno profesional, permitió articular un diálogo no sólo con los marcos normativos provenientes de Naciones Unidas, sino con el gobierno nacional de turno, pues durante la gestión presidencial de Michelle Bachelet se decidió abordar la situación de las mujeres en las Fuerzas Armadas y de Orden quienes, en general, estaban seriamente limitadas y eran objeto de un tratamiento diferenciado respecto de los hombres. Motivo por el cual, se inició en ese período la preparación de una Política de Participación de las mujeres en las Fuerzas Armadas, estableciendo criterios comunes para las distintas ramas y posibilitando la integración armónica y desarrollo profesional del personal femenino, proceso que encabezó el Ministro de Defensa Jaime Ravinet de la Fuente. En este sentido, las bases de la política que debe guiar la participación femenina en las Fuerzas Armadas serían la lgualdad de Oportunidades entre hombres y mujeres, la Integración, la Competencia profesional, la Equidad, el ejercicio del Liderazgo y el uso de la Planificación (Separata Libro de la Defensa, 2005).

No obstante, en torno a la equidad, es posible señalar que constituye aún un aspecto incipiente en el logro de esta política pública.

Tabla 3. Participación Femenina en la Institución. Cantidad y porcentaje de mujeres por grado, Oficiales.

\begin{tabular}{|l|l|l|l|l|}
\hline Grado & Hombres & Mujeres & \%Hombres & \%Mujeres \\
\hline Coronel & 297 & 1 & 99,6 & 0,4 \\
\hline Tte. Coronel & 622 & 13 & 98,0 & 2,0 \\
\hline Mayor & 605 & 35 & 95,0 & 5,0 \\
\hline Capitán & 827 & 61 & 93,0 & 7,0 \\
\hline Teniente & 604 & 51 & 92,0 & 8,0 \\
\hline Subteniente & 407 & 52 & 88,0 & 12,0 \\
\hline Alférez & 98 & 32 & 75,0 & 25,0 \\
\hline
\end{tabular}


Fuente: Elaboración propia en base a la Separata de actualización del Libro de la Defensa (2005).

Como señala el cuadro, la existencia de un Coronel femenino corresponde a un $0.4 \%$ en relación el 99,6\% del personal masculino. A pesar de la gran disparidad en términos estadísticos, representa un hecho relevante en tanto refleja la incorporación femenina a las cúpulas de poder, en donde van asumiendo una serie de responsabilidades y capacidad para participar en la toma de decisiones. Considérese las 61 mujeres que tienen el rango de Capitán, que si bien constituye el $7 \%$, manejan rangos de toma de decisiones importantes en la formación de los futuros oficiales y suboficiales del Ejército de Chile.

\section{Reflexiones Finales}

El análisis del proceso de formulación de las políticas en torno a la integración de las mujeres en el Ejército, se presenta como un proceso complejo que se desarrolló desde consideraciones basadas en la racionalidad económica hasta consideraciones políticas, las cuales no fueron excluyentes entre sí, existiendo primacía de alguna de ellas en determinado contexto socio-político.

En esa línea, es posible advertir, que desde la óptica de los actores involucrados en la formulación de las políticas públicas, la presencia de Augusto Pinochet Ugarte, Ricardo Izurieta y, posteriormente la acción conjunta de Juan Emilio Cheyre, Michelle Bachelet y Jaime Ravinet de la Fuente, configuraron contextos de intersubjetividad que representaron a las mujeres en un discurso basado en la diferencia biológica y, posteriormente, en un discurso basado en la igualdad de oportunidades y exigencias entre hombres y mujeres.

En este sentido, la hipótesis se corrobora satisfactoriamente, pues en primer lugar, la incorporación de las mujeres como parte del cuerpo profesional en la ESAFE en 1974 hasta el año 1995, se caracterizó por ser un tema que careció de políticas públicas restringiéndose al interior de la institución, primando consideraciones ético-morales representadas por el Comandante Augusto Pinochet, quien con su mirada nacionalista, gradual y jerarquizada en torno al proceso de modernización del Ejército, le asignó a la mujer un lugar en la institución según la concepción tradicional de género, en labores administrativas y logísticas. En segundo lugar, el paso de la mujer al cuerpo comando en 1995 en la Escuela Militar Bernardo O'Higgins hasta 2002 representó avances en la carrera militar y el comienzo de un diálogo con los marcos conceptuales elaborados por Naciones Unidas -nuevos escalafones y acceso al poder-. No obstante, las consideraciones ético-morales y de racionalidad económica enfocadas a una mayor eficiencia en el empleo del recurso humano siguieron primando, coincidiendo la Comandancia en Jefe de Ricardo Izurieta Caffarena con la anterior de Augusto Pinochet, 
en cierta subjetividad en la identificación del tema de la participación de las mujeres en el Ejército.

En definitiva, las intervenciones de políticas públicas en torno a las mujeres en el Ejército, se produjeron treinta años después de la incorporación de la mujer como parte del cuerpo profesional. Por lo que, en sus orígenes, la integración de las mujeres en la institución dependió en gran medida del manejo interno en el Ejército. No obstante, ese proceso no estuvo exento de las repercusiones de los contextos en los que se insertó. Así, la formulación de una política pública vía ejecutivo, se sustentó en la necesidad de atender a las demandas e intervenciones de la ONU, en atención a lo propugnado por dicho organismo diez años atrás en virtud de otorgar mayor acceso a la toma de decisiones de poder en las mujeres, bajo la lógica de la equidad de género.

En ello, la actuación política de Cheyre y su reconocimiento al tema de los Derechos Humanos, abrió la posibilidad de avanzar en la modernización en su vertiente educacional, profesionalizando al Ejército e incorporando a la mujer en todo el quehacer institucional en igualdad de condiciones y exigencias que los hombres. Situación que confluyó con el interés presidencial de Michelle Bachelet en torno a la temática de género, articulando un diálogo entre la institución militar, el gobierno de turno y los marcos conceptuales provenientes de Naciones, que dio origen a la formulación en 2005 de la Política de Integración y Participación de las mujeres en las Fuerzas Armadas y, en 2009, del Plan de Acción Nacional para la implementación de la Resolución 1325 de Naciones Unidas.

A modo de corolario, este artículo fue escrito el año 2015, su publicación en 2017 permite señalar un hecho clave, pues a partir de marzo de 2016, los alféreces Javiera Sepúlveda, Francisca Altamirano y Macarena Lobos son las primeras mujeres del Ejército de Chile en egresar de la Escuela Militar en las Armas de Combate de Infantería y Caballería Blindada, tras un año de análisis por parte del Estado Mayor del Ejército (Revista Ejército de Chile, 2017), armas que estaban vetadas para las mujeres, lo que supone un cambio cultural importante. Será labor de futuras investigaciones el análisis sobre esta nueva integración, así como la implementación y resultados de las políticas públicas a casi 10 años de formuladas.

\section{Fuentes}

Boletín Institucional, 12 de agosto de 1998, Edición año XXIV, №6708/38 Emitida por el Departamento Comunicacional del Ejército.

Clase magistral dictada por el Sr. Comandante en Jefe del Ejército, capitán General Don Augusto Pinochet Ugarte. "Ejército de Chile: Trayectoria y Futuro", Santiago, 21 de agosto de 1992. Departamento de Historia Militar. 
Circular instrucciones próximo curso E.S.A.F.E. Julio Canessa Robert. General de Brigada, Comandante de Institutos Militares. Santiago, 19. XI. 1974. Boletín Oficial del Ejército. Oscar Coddou Vivanco, Coronel, Subsecretario de Guerra.

Circular CIM ( R ) № 3751/272 de 15 de noviembre de 1974. Bol. Of. № 47 de 1974. EMGE. Depto. de Historia Militar. Archivo de Guerra. Santiago. pp. 3866-3878.

Decreto Supremo DOE. II № 317, disponible en Archivo del Ejército de Chile.

Diario La Nación, Sábado 15 de agosto de 1998.

Memorial del Ejército. Eduardo Aldunate Hermán, Teniente Coronel, Jefe del Departamento de Postgrados y Extensión de la Academia de Guerra, "La educación Superior: Agente de la Modernización del Ejército", Memorial del Ejército Edición N450, 1996, pp. 86 y 87.

Memorial del Ejército de Chile. GDE. Juan Emilio Cheyre Espinosa "Diversificación de Oportunidades y Roles en una Gran Empresa". Exposición del CJE, el 12.JUL.005., en el IV Seminario Retail sobre tendencias y oportunidades en una industria concentrada, organizado por Cámara Nacional de Comercio (CNC), en "Casa Piedra".

Memorial del Ejército de Chile N444, 1994. BGL. José Carrera Rivera. "Reflexiones". Departamento de Historia Militar.

Mujeres en la Defensa protagonistas del bicentenario, Ministerio de Defensa Nacional, marzo 2010, Academia de Guerra del Ejército.

Orden de Comando. CJE.EMGE.DPE. I/4 (R) Nº1710/02, Santiago, 17.Mayo, 1994.

Revista Armas y Servicios N81, La plena incorporación de la mujer, agosto 2003.

Separata de actualización del Libro de la Defensa 2005. 


\section{BIBLIOGRAFÍA}

Arancibia Floody Claudia y Tatiana Milstein Chateau (2002). La participación de la mujer en la Defensa Nacional y Fuerzas Armadas de Chile, Academia Nacional de Estudios Políticos y Estratégicos, Santiago: ANEPE.

Campbell, D' Ann (1993). Women in Combat: The World War II Experience in the United States, Great Britain, Germany, and the Soviet Union. The Journal of Military History.

Carreiras, Helena (1999). The Role of the Women in the Armed Forces of NATO Countries: Military Constraints and Professional Identities. Minerva: Quarterly Report on Women and the Military, XVII, (3/4), pp. $46-57$.

Castrillón Liliana y Pía Von Chrismar (2013). Mujer y Fuerzas Armadas en el contexto sudamericano: una visión desde Chile, Revista de Estudios de Seguridad y Defensa $N^{\circ} 2$, dic: ANEPE.

Costantini, Pablo (2008). La más asombrosa arma moderna del arsenal occidental. Militarización femenina en el mundo contemporáneo, Universidad Nacional de Luján, La Aljaba segunda época, volumen XII, pp. 105 - 107.

Donadio, Marcela (2010). La cuestión de género y la profesión militar; y Renata Avelar Giannini (2010). Género y fuerzas armadas en América Latina: un análisis sobre la incorporación de las mujeres en las instituciones militares de la región. En: XXIX Internacional Congress of the Latin American Studies Association (LASA), Toronto.

Gutiérrez, María Cristina y Francisca Maceratta (2010). Análisis de los antecedentes referidos a la participación de la mujer en el Ejército de Chile entre 1974 y 2005 y una breve reseña histórica de la participación de la mujer militar en las Fuerzas Armadas del mundo. Tesina (Licenciatura en Ciencias Militares). Santiago de Chile: Escuela Militar Bernardo O’Higgins Riquelme.

Hölpf, Heather J. (2008). Become a (Virile) Member: Women and the Military Body. Body and Society, 9; Sjoberg, Laura y Caron E. Gentry, Reduced to Bad Sex: Narratives of Violent Women from the Bible to the War on Terror. International Relations, 22.

Kalil Mathias, Suzeley (2005). Las mujeres llegan a los cuarteles. RESDAL.

Olavarría Gambi, Mauricio (Editor) (2010). ¿Cómo se formulan las políticas públicas en Chile. Tomo 1: La modernización de la gestión pública. Santiago, Chile: Editorial Universitaria. 
Ortiz Lazo, Claudio (2008). La modernización del Ministerio de Defensa. Ministerio de Defensa. Artículo publicado en la edición 2007 de RESDAL y actualizado para agosto de 2008.

Pérez Bravo, Claudia (2011). Una aproximación a la construcción de identidad de mujeres cadetes en la Escuela Militar del Ejército de Chile, Calidad en la Educación n³5, Departamento de Estudios Generales, Santiago de Chile: Universidad Academia de Humanismo Cristiano.

Perrot, Michelle (1997). Mujeres en la ciudad, Santiago de Chile: Editorial Andrés Bello.

Peto, Andrea (1999). Women, war and military in Eastern Europe. Minerva: Quarterly Report on Women and the Military, XVII (3/4).

Rimalt Noya (1997). Women in the Sphere of Masculinity: The Double - Edged Sword of Women's Integration in the Military. Duke Journal of Gender Law \& Policy, Vol. 14.

Segal, Mady Wechsler (1995). Women's Military Roles Cross - Nationally. Past, present and future. Gender \& Society, 9, pp. 757- 775.

Seguel Rojas, Felipe (2011). La Despinochetización del Ejército de Chile. Doctrina Cheyre: profesionalismo, modernización y pensamiento político, Tesis (Licenciatura en Historia), Santiago de Chile: Universidad de Santiago de Chile, Facultad de Humanidades.

Tellería Escobar, Loreta (2008). Mujer, Fuerza Armadas y Misiones de Paz Región Andina. Documento elaborado para el Seminario Internacional La mujer en las Fuerzas Armadas de América Latina y el Caribe: una aproximación de género a las misiones de paz, Guatemala, 11 y 12 de marzo de 2008. RESDAL.

Valenzuela, María Elena (1987). Las Mujeres en el Chile militar. Todas íbamos a ser reinas. Ediciones Chile y América - CESOC.

Villalobos, Pamela (2011). Plan de acción nacional para la implementación de la Resolución ONU 1325 Mujeres, Seguridad y Paz: La experiencia chilena. Santiago, RESDAL.

Zalaquett, Cherie (2009). Chilenas en Armas. Testimonios e historia de mujeres militares y guerrilleras subversivas, Santiago de Chile: Catalonia. 